\title{
A Hybrid Domain Degradation Feature Extraction Method for Motor Bearing Based on Distance Evaluation Technique
}

\author{
Baiyan Chen, ${ }^{1}$ Hongru Li, ${ }^{1}$ He Yu, ${ }^{1}$ and Yukui Wang ${ }^{2}$ \\ ${ }^{1}$ Mechanical Engineering College, Shijiazhuang 050003, China \\ ${ }^{2}$ Air Force Logistics College of PLA, Xuzhou 221000, China \\ Correspondence should be addressed to Hongru Li; lihr168@sohu.com
}

Received 14 November 2016; Revised 28 December 2016; Accepted 9 January 2017; Published 24 January 2017

Academic Editor: Dong Wang

Copyright (c) 2017 Baiyan Chen et al. This is an open access article distributed under the Creative Commons Attribution License, which permits unrestricted use, distribution, and reproduction in any medium, provided the original work is properly cited.

\begin{abstract}
The vibration signal of the motor bearing has strong nonstationary and nonlinear characteristics, and it is arduous to accurately recognize the degradation state of the motor bearing with traditional single time or frequency domain indexes. A hybrid domain feature extraction method based on distance evaluation technique (DET) is proposed to solve this problem. Firstly, the vibration signal of the motor bearing is decomposed by ensemble empirical mode decomposition (EEMD). The proper intrinsic mode function (IMF) component that is the most sensitive to the degradation of the motor bearing is selected according to the sensitive IMF selection algorithm based on the similarity evaluation. Then the distance evaluation factor of each characteristic parameter is calculated by the DET method. The differential method is used to extract sensitive characteristic parameters which compose the characteristic matrix. And then the extracted degradation characteristic matrix is used as the input of support vector machine (SVM) to identify the degradation state. Finally, It is demonstrated that the proposed hybrid domain feature extraction method has higher recognition accuracy and shorter recognition time by comparative analysis. The positive performance of the method is verified.
\end{abstract}

\section{Introduction}

Rolling bearing is one of the most paramount parts of the motor, which can support rotor and directly affect the operation of the motor $[1,2]$. The degraded bearing can give rise to a decline in the performance of the motor and even lead to the improper work of the entire system [3]. Therefore, it is of great significance and practical value to extract degradation feature. As plenty of state information is hidden in the motor bearing vibration signal, the analysis of vibration signal is one of the main methods to extract degradation feature. The degraded bearing itself easily trigger nonconcentric fault of the motor stator and rotor. Consequently, the motor bearing vibration signals show strong nonstationary and nonlinear characteristics $[4,5]$. The difficulty and complexity of extracting effective degradation feature is greatly increased. Degradation state recognition of the bearing consists of three steps, including information acquisition, feature extraction, and pattern recognition [6, 7]. Accordingly, it is the key of degradation state recognition to extract degradation feature accurately and effectively.
EEMD method is especially applicable to the analysis of nonstationary and nonlinear signals, and the method has a wide range of applications in many fields [8, 9]. Žvokelj et al. [10] utilized EEMD method to process acoustic emission (AE) signal of slewing bearing, and the mixed domain fault feature of each IMF component was extracted through principal component analysis (PCA). Lei et al. [11] combined EEMD and wavelet neural network (WNN) to achieve locomotive roller bearing fault diagnosis. Jiang et al. [8] proposed an improved EEMD method with multiwavelet packet, and it was applied to rotating machinery multifault diagnosis. It is bound to be reflected in a certain IMF component when the bearing fault appears. The degradation characteristics will be more obvious after the amplification of IMF. Therefore, it can achieve a better feature extraction effect based on EEMD. Single domain feature is arduous to fully and accurately describe the different degrees of degradation in complex mechanical systems [12]. A method based on a hybrid domain feature extraction is proposed to make full use of time domain, frequency domain, and time-frequency domain characteristic information. 
The feature fusion method can make full use of the feature information of multiple domains, which can reflect the degradation state of the equipment more comprehensively. Yu [13] fused multiple domain features with locality preserving projections (LPP) for bearing performance degradation assessment. Chen et al. [14] utilized Local tangent space alignments (LTSA) to fuse multiple domain features for rolling bearing multifault diagnosis study. However, the fused feature does not enjoy a clear physical meaning, and it can not explain the specific performance of the feature as the degenerative state changes. The extracted feature values often appear in the following two conditions [15]. One is the characteristic parameter which has no relation with the classified target, and the other is the redundant characteristic parameter which has high correlation with other characteristic parameters. Feature selection is to select the most effective features from a set of features to reduce the dimension of the feature space [16]. Compared with feature fusion method, the advantage of using feature selection methods for dimension reduction is that the feature is not transformed and still maintains the original physical meaning [17-19]. As a classical algorithm for feature selection, DET can reduce the time of degradation state recognition and ensure the accuracy of recognition at the same time.

Based on the above analysis, motor bearing hybrid domain degradation feature extraction based upon EEMD and DET method is proposed in this paper. Firstly, EEMD method is used to decompose the vibration signal of the motor bearing, and the sensitive components are selected according to the sensitive factors. Hybrid domain degradation features are constructed, and sensitive components are selected by DET method. Finally, the degradation state recognition rate obtained by SVM is used to verify the accuracy of the feature extraction.

The paper is organized as follows: Section 2 presents the EEMD method. And the selection of the sensitive IMF component is also given in this section. Section 3 presents a mathematical analysis in detail to select the most effective features based on DET method. Section 4 presents a favorable discussion and analysis of the experimental results by comparing the proposed method with single domain degradation feature extraction and high dimension degradation feature extraction. Finally, our conclusions are provided in Section 5.

\section{Algorithm of EEMD}

2.1. EEMD Method. A new noise-assisted data analysis method was proposed to overcome the problem of mode mixing in EMD [20]. It is EEMD which defines the true IMF components as the mean of an ensemble of trials. Each trial consists of the decomposition results of the signal adding a white noise of finite amplitude.

The algorithm is described as follows [21].

(1) Add white noise sequence to the signal $x(t)$.

$$
x^{\prime}(t)=x(t)+n(t)
$$

where $x^{\prime}(t)$ represents the noise-added signal and $n(t)$ indicates the added white noise.
(2) $x^{\prime}(t)$ is decomposed into IMFs according to the EMD method.

(3) Repeat steps (1) and (2) by adding a different white noise sequence.

(4) The average value of the corresponding IMFs is obtained.

2.2. Selection Algorithm Based on the Similarity Evaluation. The signal is decomposed into a group of IMFs by EEMD. A portion of the IMFs are sensitive components closely related to the degradation state, while the others are degenerate independent or noise interfering components. Consequently, it is essential to choose sensitive IMFs which are closely related to the bearing degradation before evaluating the sensitivity of each characteristic parameter. It can improve the accuracy of the degradation feature extraction by ignoring other irrelevant IMFs [22]. A sensitive IMF selection algorithm based on similarity evaluation is adopted in this paper.

The specific algorithm of sensitive IMF selection algorithm [23] is as follows.

(1) Calculate correlation coefficients $u_{n}$ between signal $x(t)$ and its $\mathrm{IMF}_{n}$, where $n=1,2, \ldots, N$.

(2) Calculate correlation coefficients $\beta_{n}$ between the $\mathrm{IMF}_{n}$ of signal $x(t)$ and signal $x(t)$ collected under normal operating conditions.

(3) The correlation coefficient of bearing degradation is defined as

$$
\eta_{n}=\beta_{n}-u_{n}
$$

where $n=1,2, \ldots, N$.

(4) Define and calculate IMFs degradation sensitivity factor $\lambda_{n}$ as

$$
\lambda_{n}=\frac{\eta_{n}-\min (\eta)}{\max (\eta)-\min (\eta)}
$$

where $n=1,2, \ldots, N$.

(5) All IMFs are sorted in accordance with the sensitive factor from large to small order, and the difference of the sensitivity factor of two adjacent IMFs is calculated. The index of the corresponding maximum difference $n$ is found. Then the former $n$ IMFs are degradation feature sensitive IMF components.

IMF sensitivity factor not only considers the similarity between IMF and the signal itself but also takes into account the similarity between the IMF and the normal signal. The IMFs containing more fault information are more similar to the origin signal. The information that is not related to the fault in IMF can be evaluated through calculating the correlation coefficient between the IMF component and the normal signal. Consequently, the method is more accurate than only calculating the correlation coefficient between IMF component and origin signal in selecting the sensitive IMF components. 
TABLE 1: Hybrid domain features.

\begin{tabular}{lr}
\hline Peak value & $x_{p}=\left|x_{i}\right|_{\max }$ \\
Root mean square amplitude & $X_{r}=\left(\frac{1}{N} \sum_{i=1}^{N} x_{i}^{1 / 2}\right)^{2}$ \\
Absolute mean value & $\mu_{|x|}=\frac{1}{N} \sum_{i=1}^{N}\left|x_{i}\right|$ \\
Root mean square value & $X_{\mathrm{rms}}=\sqrt{\frac{1}{N} \sum_{i=1}^{N} x_{i}^{2}}$ \\
Peak index & $\mathrm{C}=\frac{X_{p}}{X_{\mathrm{rms}}}$ \\
Waveform index & $I_{f}=\frac{X_{p}}{\mu_{|x|}}$ \\
Clearance factor & $\mathrm{CL}=\frac{X_{p}}{X_{r}}$ \\
Center of gravity frequency & $\mathrm{FC}=\frac{\sum_{i=1}^{N} \overline{x_{i}} \cdot x_{i}}{2 \pi \sum_{i=1}^{N} x_{i}^{2}}$ \\
Mean square frequency & $\mathrm{MSF}=\frac{\sum_{i=1}^{N} \bar{x}_{i}^{2}}{4 \pi^{2} \sum_{i=1}^{N} x_{i}^{2}}$ \\
Root mean square frequency & $\mathrm{RMSF}=\sqrt{\mathrm{MSF}}$ \\
Frequency variance & $H_{p}=\frac{\sum_{j=1}^{k} P_{j} \ln \left(P_{j}\right)}{\ln (m !)}$ \\
\hline
\end{tabular}

\section{DET Method and Degradation Feature Extraction}

3.1. Characteristic Parameters of Motor Bearing. IMF sensitive component is regarded as the research object after EEMD decomposition. 12 characteristic parameters existing in the time domain, the frequency domain, and the time-frequency domain are extracted from each IMF component, which are conducive to status identification for motor bearings. Time domain parameters include peak value, root mean square amplitude, absolute mean value, root mean square value, peak index, waveform index, and clearance factor. Frequency domain parameters include center of gravity frequency, mean square frequency, root mean square frequency, and frequency variance. Permutation entropy (PE) is determined as a characteristic parameter based on complexity, whose embedding dimension $m=6$ and the time delay $\tau=1$ [24]. This paper proposes to use hybrid features displayed in Table 1.

$x_{i}$ represents the vibration signal. $\overline{x_{i}}=\left(x_{i}-x_{i-1}\right)$. $F_{s} ; F_{s}$ represents sampling frequency. $N$ represents sampling points. $P_{j}$ is used to denote the probability distribution of each symbol sequences.

3.2. Calculation of Distance Evaluation Factor. DET is a kind of feature evaluation technology based on inner class distance [25]. The evaluation principle can be described as that the same kind of inner characteristic distance is the smallest, and the distance between two different classes is the biggest.
Features complying with this principle are considered to be sensitive features.

The specific implementation process of the evaluation method is as follows [25].

Assume there are a total of $N$ species of degradation states of the motor bearing and $M$ samples are selected for each state and $C$ parameters are extracted from each sample. An obtained characteristic matrix is $\left\{f_{n, m, c}, n=1,2, \ldots, N ; m=\right.$ $1,2, \ldots, M ; c=1,2, \ldots, C\} . f_{n, m, c}$ represents $c$ th characteristic of $m$ th sample of $n$th degradation state.

(1) The inner class average Euclidean distance of the motor bearing in the same state is calculated. (Distance is Euclidean distance without special instructions.)

$$
D_{n, c}=\frac{1}{M \times(M-1)} \sum_{\substack{l, m=1 \\ l \neq m}}^{M}\left|f_{m, n, c}-f_{l, n, c}\right| .
$$

The average distance between the classes of $N$ species is obtained.

$$
D_{c}^{(w)}=\frac{1}{N} \sum_{n=1}^{N} D_{n, c}
$$

(2) The difference factor of the inner class distance in the same condition is defined by analyzing the frequency spectrum of the vibration signal.

$$
v_{c}^{(w)}=\frac{\max \left(D_{n, c}\right)}{\min \left(D_{n, c}\right)} .
$$

(3) The average value of each feature of all samples in the same class is calculated.

$$
u_{n, c}=\frac{1}{M} \sum_{m=1}^{M} f_{m, n, c} .
$$

The average distance between different states of each feature is obtained.

$$
D_{c}^{(b)}=\frac{1}{N \times(N-1)} \sum_{\substack{n, e=1 \\ n \neq e}}^{N}\left|u_{n, c}-u_{e, c}\right| .
$$

(4) The status of the motor bearing is examined by the variation of the characteristic parameter. The difference between class distances is defined as follows:

$$
v_{c}^{(b)}=\frac{\max \left(\left|u_{n, c}-u_{e, c}\right|\right)}{\min \left(\left|u_{n, c}-u_{e, c}\right|\right)},
$$

where $n, e=1,2, \ldots, N ; n \neq e$.

(5) Weighting factors are calculated.

$$
\lambda_{c}=\frac{1}{v_{c}^{(w)} / \max \left(\nu_{c}^{(w)}\right)+v_{c}^{(b)} / \max \left(v_{c}^{(b)}\right)} .
$$

(6) The ratio of all parameters average distance in different states and average distance in the same state are calculated considering the influence of weighting factors.

$$
\alpha_{c}=\lambda_{c} \frac{D_{c}^{(b)}}{D_{c}^{(w)}} .
$$


(7) The results obtained are normalized.

$$
\beta_{c}=\frac{\alpha_{c}}{\max \left(\alpha_{c}\right)} .
$$

$\beta_{c}$ is the distance evaluation factor of characteristic parameters. It reacts to the sensitivity of each characteristic parameter directly.

3.3. Selection of Sensitive Degradation Features Based on Distance Evaluation Factor. It is a knotty problem how to make use of the distance evaluation factor to select the sensitive degradation features to reduce the recognition time. Currently, there is no accepted standard for the selection of thresholds [26]. Quite a few scholars have been inspired by the Wrapper method. They used the classifier to determine the threshold value of the method to screen the sensitive features [25]. However, the method has the shortcomings of high time complexity, large calculation, and poor generalization ability. In addition, a number of tests are needed [19]. The differential method is utilized to screen sensitive degradation characteristics in this paper. Compared with the method of determining the threshold value according to the recognition rate of classifier, the calculation is smaller, and the degradation features can be selected without the intervention of the classifier. Firstly, the characteristic parameters are extracted as degradation state and the operating condition (five kinds of degradation States, four operating conditions) synchronously change and the operating conditions (four conditions, the inner race degradation 0.007 inch) barely change. The distance evaluation factor of each parameter in the two cases is determined by calculating the distance evaluation factor. The difference between the distance evaluation factor of the degradation state and the operating condition at the same time is subtracted from the distance evaluation factor when the condition is changed. The difference between distance evaluation factor for degradation state and operating condition simultaneous changing and distance evaluation factor for only operating conditions changing is obtained. For $D$ kinds of different conditions, $\beta_{c d}$ according to the above method can be obtained. The difference between $\beta_{c}$ and $\beta_{c d}$ is $\chi_{c}$. The characteristic parameters are more sensitive to the change of the state of the motor bearing as $\chi_{c}$ is greater. The characteristic parameters of $\chi_{c}>0$ are selected as the sensitive parameters that exclude the influence of operating condition.

3.4. Support Vector Machine (SVM). Support vector machine (SVM) is an effective computational learning method presented by Vapnik [27], which specializes for a smaller number of samples for training. The basic principle of SVM can be illustrated in a two-dimensional way as represented in Figure 1. It demonstrates the classification of a series of points for two different classes, Class 1 and Class 2. The SVM tries to calculate the distance between the boundary and the nearest data point in each class maximal. The nearest data points are regarded as support vectors.

SVM classification function output is a linear combination of intermediate nodes, and each intermediate node

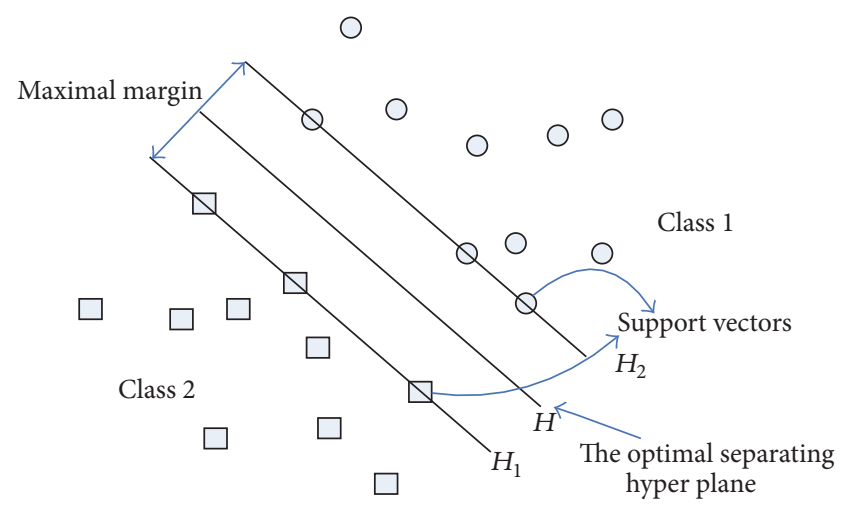

FIGURE 1: The optimized separating hyper plane in classification.

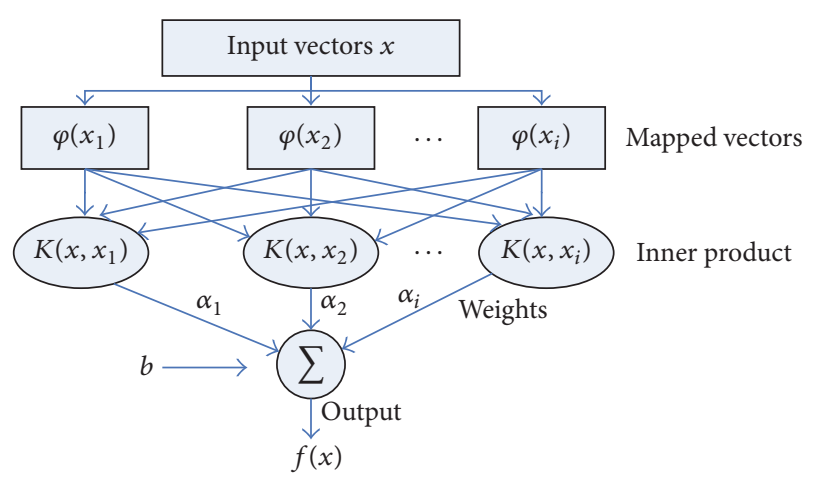

FIgURE 2: The architecture of SVM.

corresponds to a support vector. The architecture of SVM is manifested in Figure 2.

Consider a training sample set, $S=\left\{\left(x_{i}, y_{i}\right) \mid x_{i} \in\right.$ $\left.R^{N}, y_{i} \in\{-1,1\}, i=1,2, \ldots, l\right\}$, where $x_{i}$ is an input vector and $y_{i}$ is a label of $x_{i}$. The general form of linear discriminant function in $n$-dimensional space is $g(x)=(w \cdot x)+b$. The data in the set can be correctly classified by the optimized hyperplane. SVM turns to the following dual optimization problem for classification problem:

$$
\begin{array}{ll}
\operatorname{minimize} & \frac{1}{2} w^{T} w+C \sum_{i=1}^{n} \zeta_{i} \\
\text { subject to } & y_{i}\left[\left(w \cdot x_{i}+b\right)\right]+\zeta_{i} \geq 1,
\end{array}
$$

where $w$ and $b$ represent undetermined coefficient. $C$ represents penalty factor. $\zeta_{i}$ represents relaxation factor.

The final result is a discrimination function $f(x)$ conveniently expressed as a function of the data in the lower dimensional feature space:

$$
f(x)=\operatorname{sgn}\left(\sum_{i=1}^{n} \alpha_{i} y_{i} K\left(x_{i}, x\right)+b\right) .
$$

Kernel function can avoid complex operation in high dimensional feature space. The most widely adopted kernel 


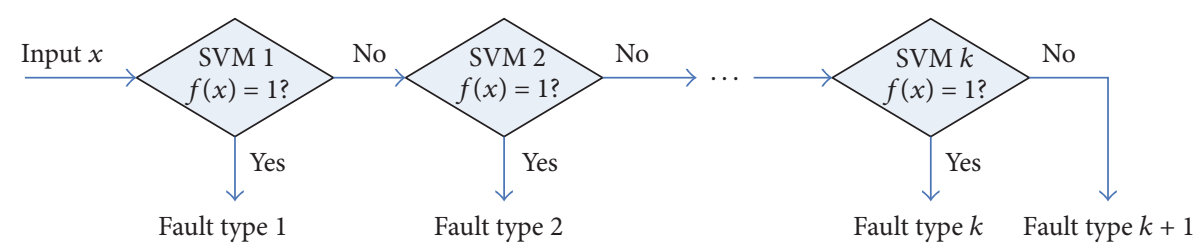

FIGURE 3: "One to others" multiclass SVM classifier.

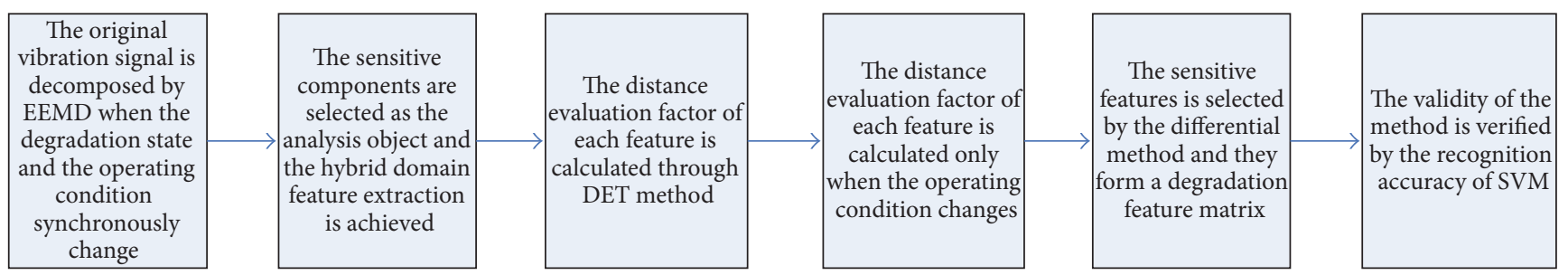

FIGURE 4: Motor bearing degradation feature extraction process based on DET.

function is the radial basis function (RBF), and it is defined as follows:

$$
K\left(x, x_{i}\right)=\exp \left(-\frac{\left\|x-x_{i}\right\|^{2}}{2 \sigma^{2}}\right) .
$$

SVM is mainly used as "one to one" binary classifier and "one to others" multiclass classifier. SVM is utilized to identify the different degradation states of the motor bearings. Accordingly, multiclass classifier is determined in this paper. A multiclass classifier consists of an ocean of binary classifier. The process of the multiclass SVM is described in Figure 3.

\subsection{Hybrid Domain Degradation Feature Extraction Method} Based on DET. The process of motor bearing degradation feature extraction based on DET is shown in Figure 4.

(1) The original vibration signal is decomposed by EEMD when the degradation states and the operating conditions synchronously change, and the sensitive IMF components are selected as the analysis object according to the similarity evaluation algorithm.

(2) For each sensitive IMF component, 12 characteristic parameters are extracted to form a hybrid domain feature matrix.

(3) The distance evaluation factors of characteristic parameters are obtained according to the DET method.

(4) Repeat steps (1)-(3) in a special degradation state, only when the operating conditions change.

(5) According to the difference method, the sensitive features are selected to form the degradation feature matrix.

(6) Finally, the effectiveness of the proposed method is verified by SVM.

\section{Experimental Results}

4.1. Experiment Data Sources. The data of deep groove ball bearings of 6205-2RS JEM SKF from the bearing data center of Case Western Reserve University is used as the experimental raw data in our study [28]. The test bed is manifested in
Figure 5. The test rig consists of a $2 \mathrm{Hp}$ (horsepower) motor, a torque converter, a dynamometer, and the control circuit. The vibration signals, including the normal, inner race fault, outer race fault, and rolling element fault signal, are collected by an acceleration sensor installed on the magnetic base shell with a sampling frequency of $12 \mathrm{kHz}$, and sampling points are 1024 . In this paper, the degradation states of inner race serve as an example for analysis, and four operating conditions exist in each state. For each fault mode, data are collected under four operating conditions corresponding to motor speeds of 1730 , 1750,1772 , and $1797 \mathrm{r} / \mathrm{min}$. Inner race fault diameter is $0.007^{\prime \prime}$, $0.014^{\prime \prime}, 0.021^{\prime \prime}$, and $0.028^{\prime \prime}$. The normal state can be seen as a special degradation state, and there are five kinds of degradation states. The vibration signal of the five degradation states and their frequency spectrum are shown in Figure 6.

4.2. Degradation Feature Extraction Based on DET. First of all, it is analyzed under the condition of five kinds of degradation states, and four kinds of working conditions exist in each state.

The collected signal is decomposed by EEMD method, and the sensitive components are selected based on the similarity assessment of sensitive IMF selection algorithm. The results are shown in Figure 7. Each signal can be decomposed to get the first two IMF components for the sensitive IMF components. The sensitive components of the five degradation states are manifested in Figure 8. Feature extraction is performed by selecting the IMF components which contain the main degradation state information. In this paper, 12 mixed domain parameters of the IMF components are extracted as the characteristic value, including peak value, root mean square amplitude, absolute mean value, root mean square value, peak index, waveform index, clearance factor, center of gravity frequency, mean square frequency, root mean square frequency, frequency variance, and permutation entropy. Therefore, 24 characteristic values can be extracted for each signal. The characteristics of the IMF1 component values are $t_{1} \sim t_{12}$, and characteristics of IMF2 component values are $t_{13} \sim t_{24}$. Then, the degradation features are extracted 

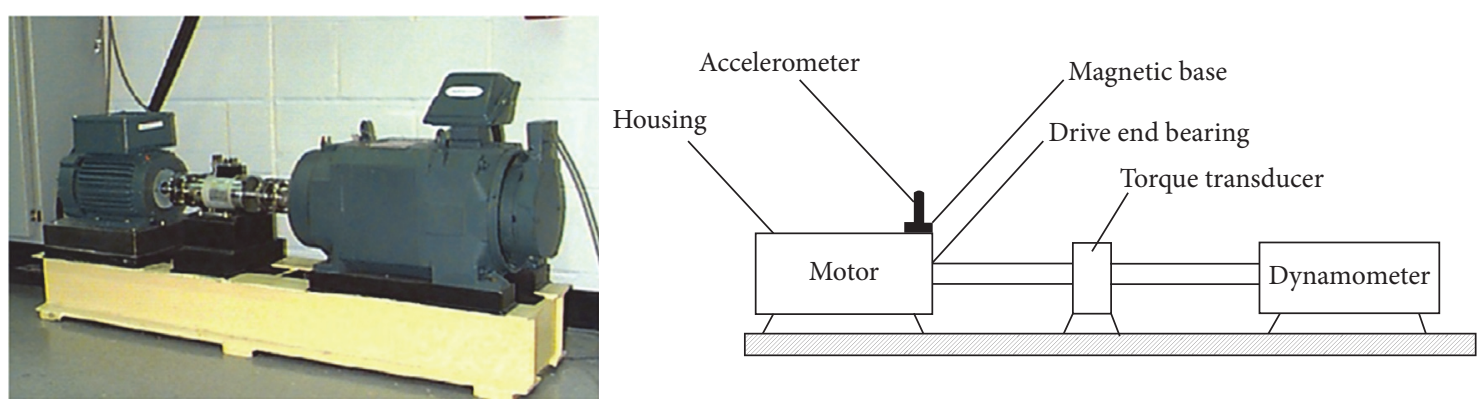

FIGURE 5: The bearing experimental system.
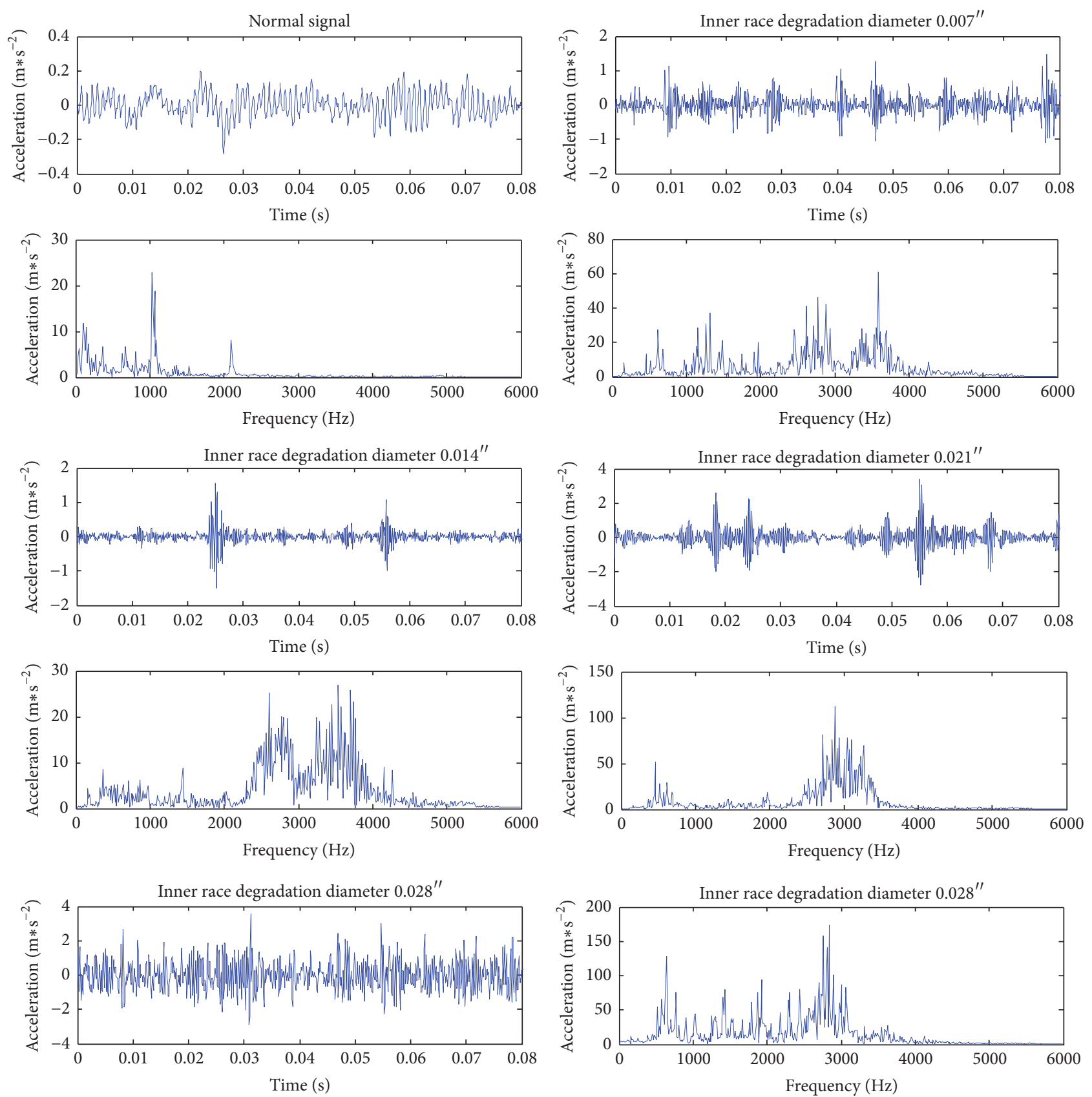

FIGURE 6: Time domain and frequency spectrum of five-degradation state vibration signal of the motor bearing. 


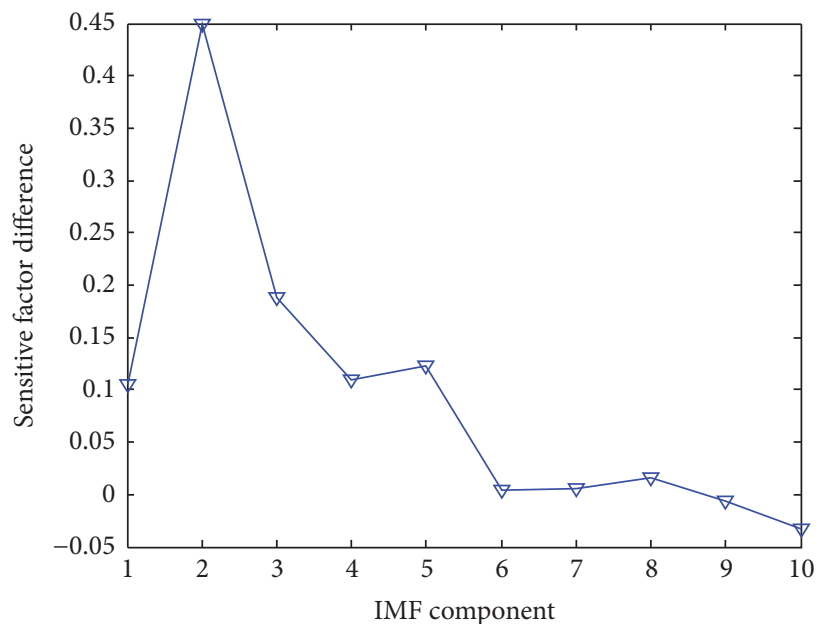

(a) Inner race degradation diameter $0.007^{\prime \prime}$

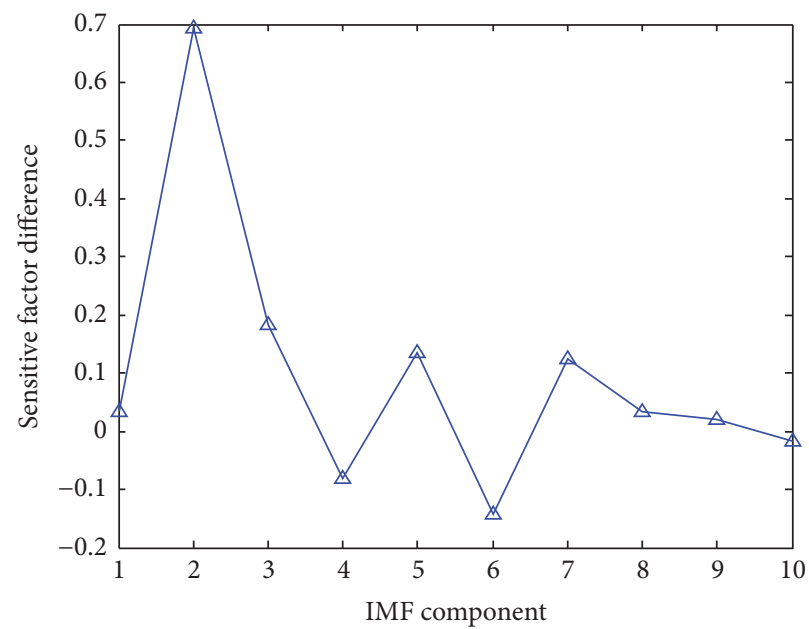

(c) Inner race degradation diameter $0.021^{\prime \prime}$

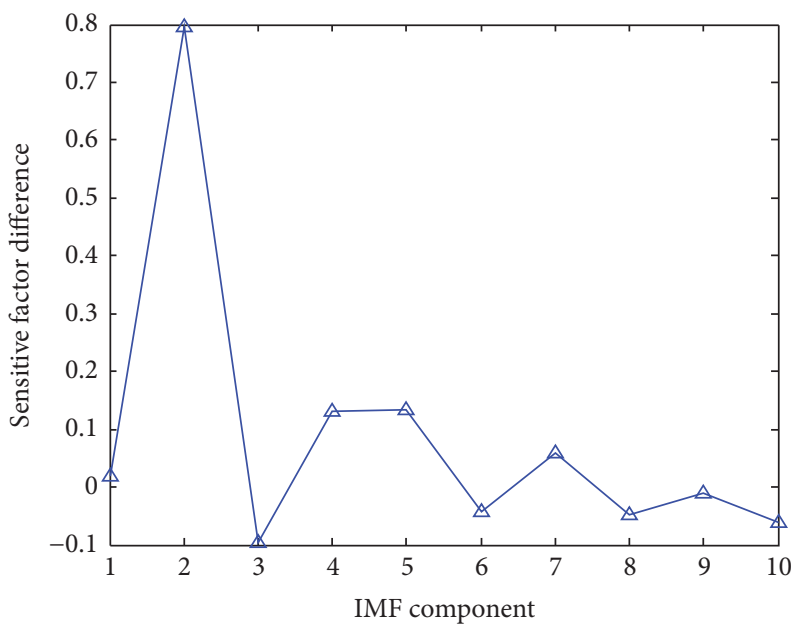

(b) Inner race degradation diameter $0.014^{\prime \prime}$

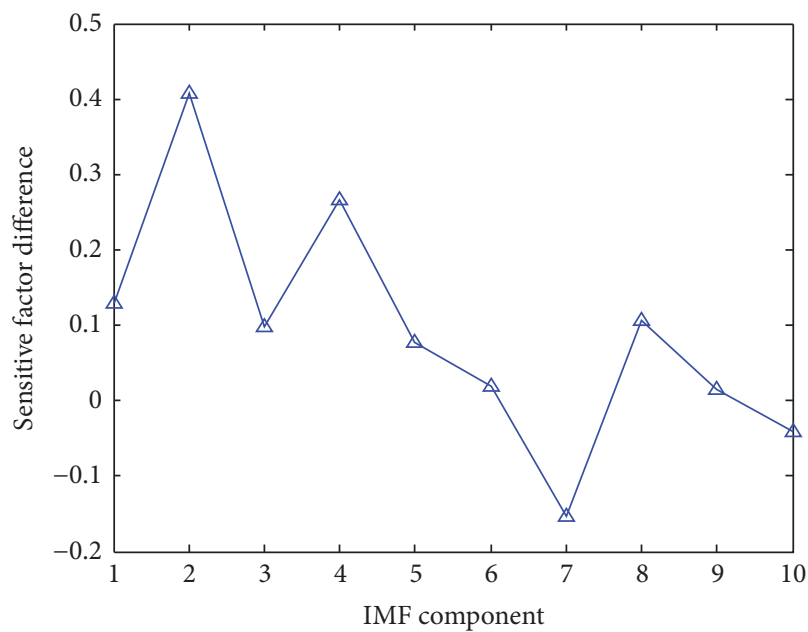

(d) Inner race degradation diameter $0.028^{\prime \prime}$

FIGURE 7: The sensitive IMF components based on similarity evaluation.

through the DET method. Before feature selection, the sensitivity to class of each characteristic parameter that is the distance evaluation factor $\beta_{c}$ has been calculated. In a specific degradation state, the distance evaluation factor $\beta_{c d}$ is obtained in accordance with the same method only when four kinds of operating conditions change. The differences between $\beta_{c}$ and $\beta_{c d}$ named $\chi_{c}$ are demonstrated in Figure 9. The positive direction of $y$-axis indicates that it is sensitive to the change of degradation state and is not sensitive to the change of operating condition. It is contrary to the negative direction of $y$-axis. The sensitive characteristic values are selected by the differential method, and the degradation characteristic matrix is further formed. Distance evaluation factors and their differences are displayed in Table 2.

The selected feature parameters, that is, $\left[t_{20}, t_{18}, t_{17}, t_{16}\right.$, $\left.t_{19}, t_{23}, t_{24}, t_{9}, t_{11}, t_{13}\right]$, can be clearly seen according to Figure 9. Degradation feature is reduced from 24 dimensions to 10 dimensions and the physical meaning of the original feature is maintained.
4.3. The Effectiveness of the Degradation Feature Extraction. In order to illustrate the effectiveness of the proposed method, SVM method is used to verify the effectiveness of the degradation feature extraction. The recognition accuracy of SVM output and the recognition time of SVM are used as the basis for judgment [4]. Fifty groups of samples are trained for each degenerate state, and fifty groups of samples are tested. The RBF kernel function is selected as SVM kernel function, and its classification performance is better than other types of nuclear function [29]. The penalty parameter $C$ and kernel function parameter $g$ are selected and the particle swarm optimization (PSO) algorithm is used to optimize the process [30]. The number of particles is set to 20 per degenerate state. Iteration maximal number is set to 200 . The range of $C$ is set from 0.1 to 100 , and the range of $g$ is set from 0.01 to 1000 . The highest recognition accuracy is taken as the optimization target. The fitness curve of parameter optimization is manifested in Figure 10 (since the iteration of the twelfth generation has been convergent, only the first fifty generations are listed). The time of PSO searching for 

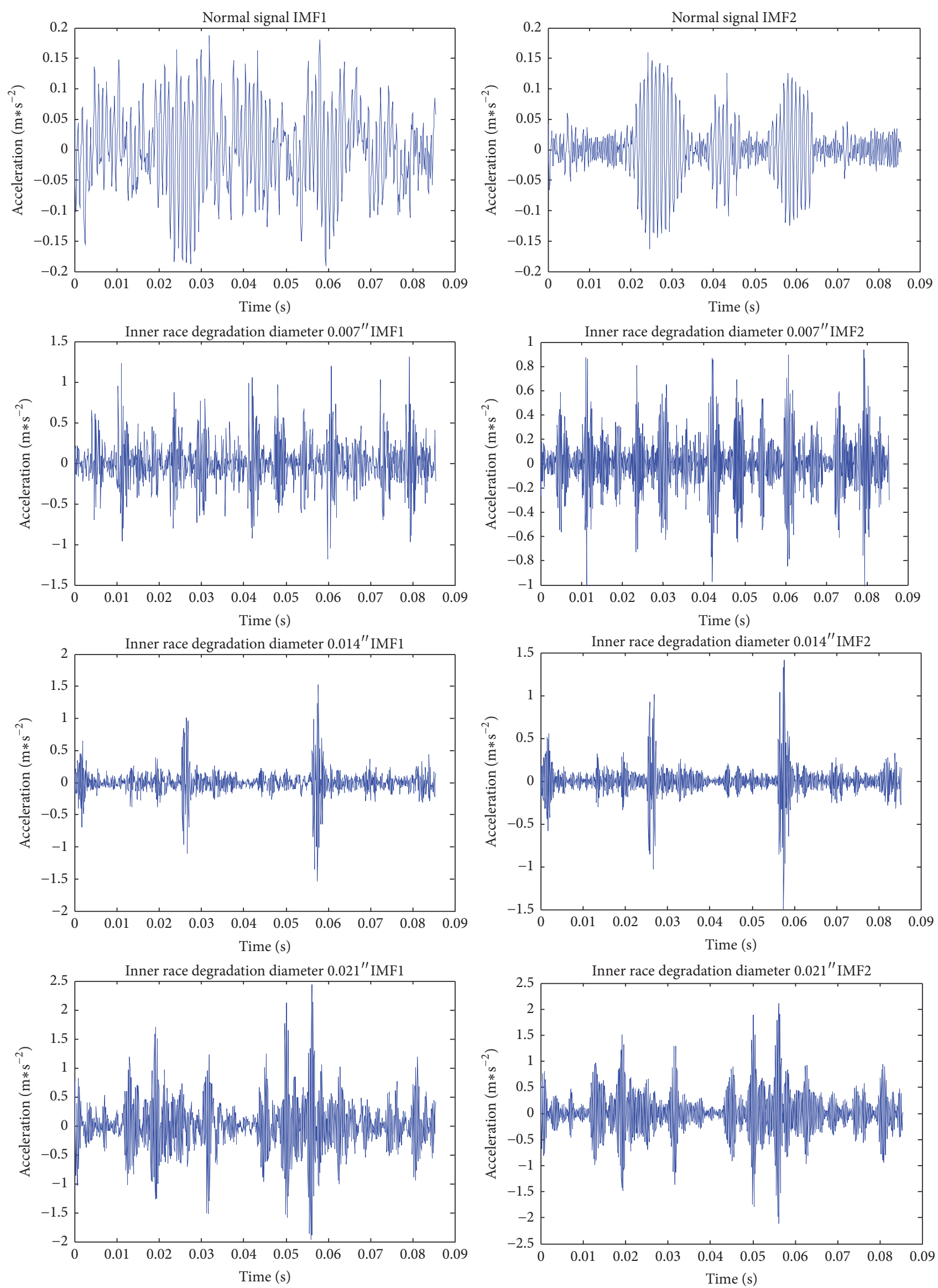

Figure 8: Continued. 

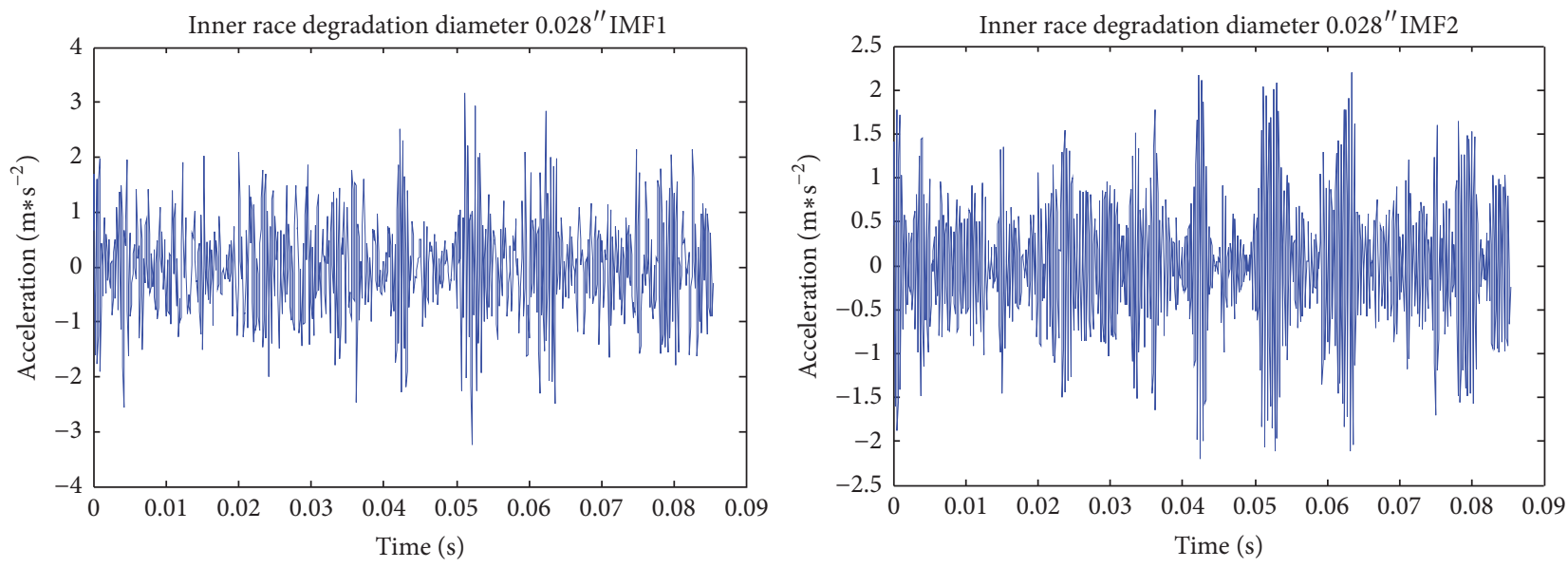

FIGURE 8: The sensitive IMF components of the five degradation states.

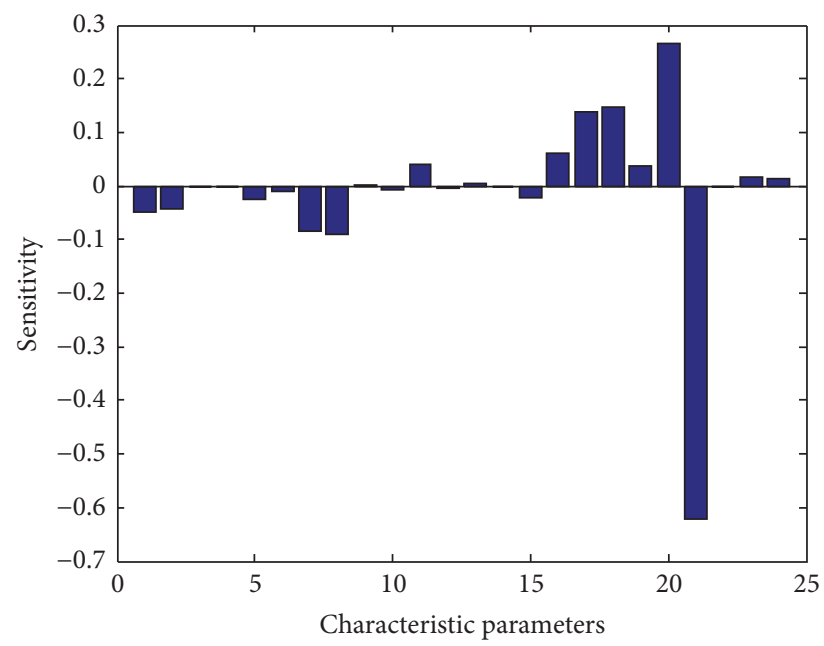

FIGURE 9: Sensitivity of each characteristic parameter.

optimized parameter is 2.852146 s (operating platform for R201lb Matlab; the main computer is configured as follows: CPU Intel(R) Core(TM) i5-4590 $3.30 \mathrm{GHz}$, memory $4 \mathrm{G}$ ). The result of particle seeking optimal parameters in twodimensional space is that the optimal penalty parameter $C=$ 4 and the kernel function parameter $g=0.125$.

The identification result is demonstrated in Figure 11. Test sample labels 1, 2, 3, 4, and 5, respectively, represent five degradation states. The final recognition rate reaches $99.6 \%$ and the recognition time is $9.902657 \mathrm{~s}$. The results indicate that most of the test data are identified correctly. The effectiveness of the proposed method is verified.

4.4. Comparative Analysis. In order to further verify the effect of the degradation feature extraction based on DET, we use the single domain feature extraction and high dimension feature extraction to compare the proposed feature extraction. The result of degradation state recognition rate and the recognition time is displayed in Table 3.
TABLE 2: Distance evaluation factors and their differences between $\beta_{c}$ and $\beta_{c d}$.

\begin{tabular}{lccc}
\hline Feature parameter & $\beta_{c}$ & $\beta_{c d}$ & $\chi_{c}$ \\
\hline$t 1$ & 0.0315 & 0.0811 & -0.0496 \\
$t 2$ & 0.0409 & 0.0849 & -0.0440 \\
$t 3$ & $4.6864 e-04$ & 0.0030 & -0.0026 \\
$t 4$ & 0.0023 & 0.0028 & $-4.6605 e-04$ \\
$t 5$ & 0.0064 & 0.0321 & -0.0258 \\
$t 6$ & 0.0174 & 0.0281 & -0.0107 \\
$t 7$ & 0.0111 & 0.0951 & -0.0840 \\
$t 8$ & 0.0037 & 0.0927 & -0.0890 \\
$t 9$ & 0.0114 & 0.0082 & 0.0032 \\
$t 10$ & 0.0018 & 0.0082 & -0.0064 \\
$t 11$ & 0.0452 & 0.0037 & 0.0415 \\
$t 12$ & 0.0044 & 0.0079 & -0.0035 \\
$t 13$ & 0.0118 & 0.0069 & 0.0050 \\
$t 14$ & 0.0038 & 0.0062 & -0.0024 \\
$t 15$ & 0.0765 & 0.0972 & -0.0206 \\
$t 16$ & 0.1938 & 0.1332 & 0.0606 \\
$t 17$ & 0.3834 & 0.2453 & 0.1381 \\
$t 18$ & 0.4835 & 0.3354 & 0.1482 \\
$t 19$ & 0.3104 & 0.2743 & 0.0361 \\
$t 20$ & 0.5367 & 0.2717 & 0.2650 \\
$t 21$ & 0.2700 & 0.8912 & -0.6212 \\
$t 22$ & 1 & 1 & 0 \\
$t 23$ & 0.0257 & 0.0101 & 0.0156 \\
$t 24$ & 0.0257 & 0.0119 & 0.0138 \\
\hline
\end{tabular}

Firstly, single domain feature extraction is performed for sensitive IMF components. According to Table 1, the root mean square frequency of IMF2 is the most sensitive. This feature is selected as the degradation feature, and the effectiveness of the feature extraction is verified by SVM. The degradation state recognition result is shown in Figure 12.

Secondly, the feature extraction of high dimension degradation is performed for sensitive IMF components, that is, 
TABLE 3: Recognition rate and recognition time.

\begin{tabular}{lcc}
\hline Method & $\begin{array}{c}\text { Recognition } \\
\text { rate }\end{array}$ & $\begin{array}{c}\text { Recognition } \\
\text { time }\end{array}$ \\
\hline The single domain feature extraction & $98.4 \%$ & $8.854626 \mathrm{~s}$ \\
High dimension feature extraction & $99.2 \%$ & $19.293885 \mathrm{~s}$ \\
The proposed method & $99.6 \%$ & $9.902657 \mathrm{~s}$ \\
\hline
\end{tabular}

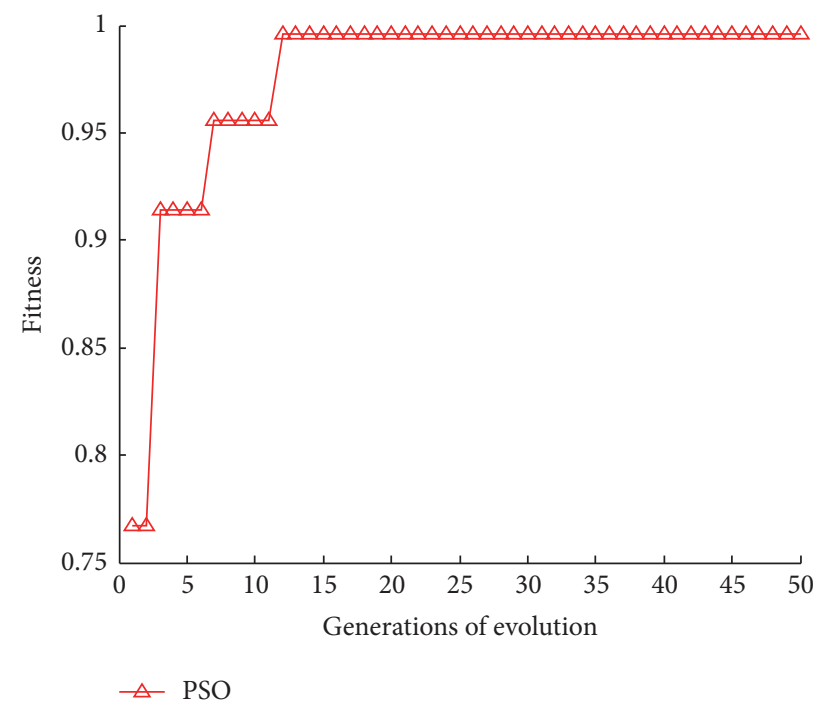

FIGURE 10: The fitness curve of parameter optimization.

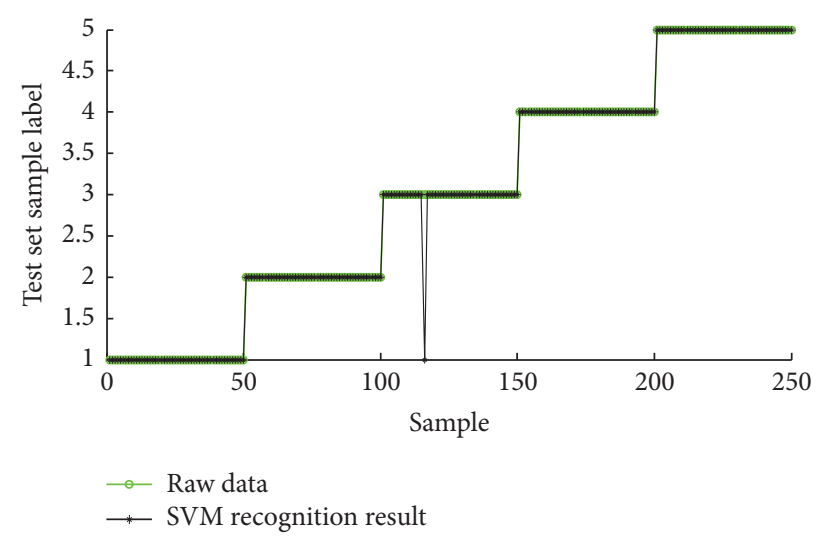

FIGURE 11: Degradation state recognition results.

screening sensitive features without the method of DET. 24 characteristics are used to form a degeneration feature matrix, and SVM is used to verify the effectiveness of the feature extraction. The recognition rate is manifested in Figure 13.

Through the comparative analysis, we can draw the following conclusions: the proposed method can extract the degradation state information of motor bearings more effectively. The redundant features of the motor bearing degradation state can be removed, and the degradation feature can be extracted more accurately.

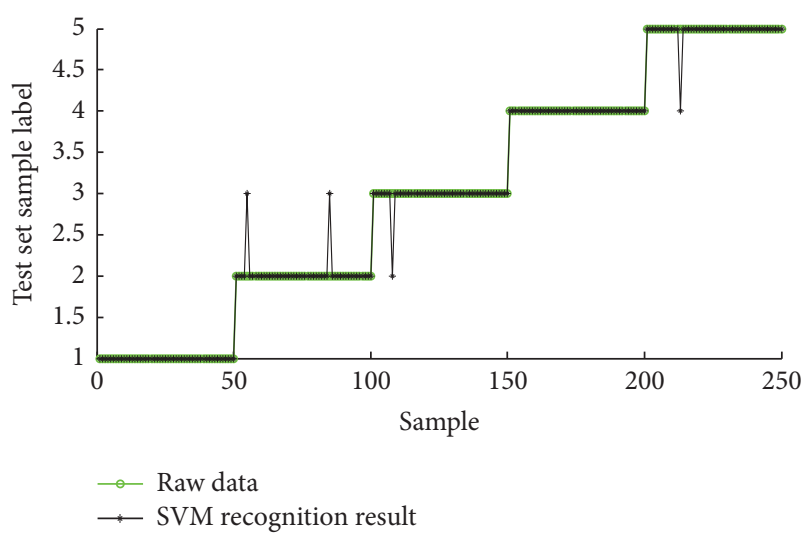

FIGURE 12: Degradation state recognition results for single domain degradation feature.

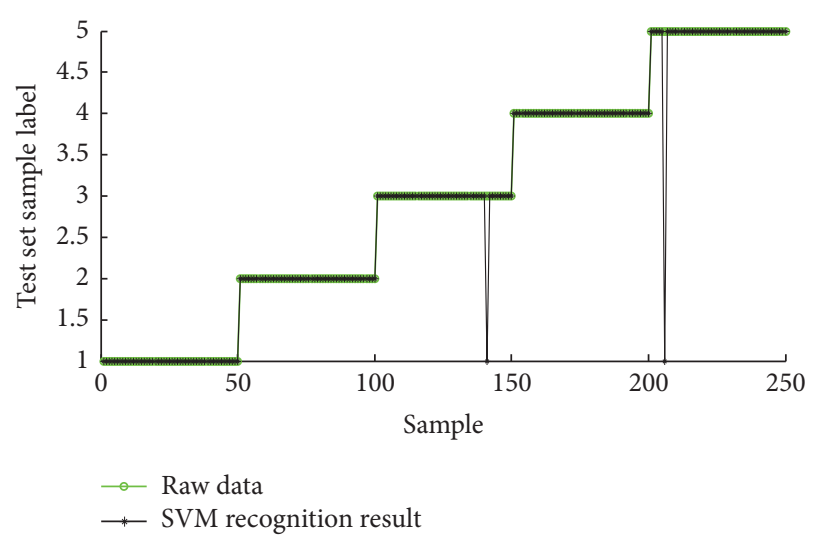

FIGURE 13: Degradation state recognition results for high dimension degradation feature.

\section{Conclusions}

In this paper, a hybrid domain degradation feature extraction method based on DET is proposed to extract the degradation characteristics. Finally, the recognition rate of SVM output demonstrates the validity and accuracy of the feature extraction method. Through the analysis, we can get the following conclusions.

(1) The hybrid domain feature is more comprehensive than the single domain feature, which can reflect the degradation state of the bearing accurately. The proposed method can be overwhelmingly accurate to select the appropriate characteristic parameters of the motor bearing degradation state. It can achieve the degradation feature extraction more accurately.

(2) The DET method can remove redundant features and unrelated features effectively, which can maintain the physical meaning of the original feature parameters and shorten the recognition time.

(3) The sensitive feature selection is related to the quality of the feature extraction. The degradation feature is extracted through the proposed method, which is applied to the actual vibration signal of the motor bearing. The recognition rate 
can reach $99.6 \%$, and the result indicates the validity and feasibility of the proposed method.

\section{Competing Interests}

The authors declare that they have no competing interests.

\section{Acknowledgments}

The authors are grateful to Case Western Reserve University. This project is supported by National Natural Science Foundation of China (Grant no. 51541506).

\section{References}

[1] Z. Li, J. Zhu, X. Shen, C. Zhang, and J. Guo, "Fault diagnosis of motor bearing based on the Bayesian network," Procedia Engineering, vol. 16, pp. 18-26, 2011.

[2] B. Wang, H.-R. Li, and B.-H. Xu, "Motor bearing forecast feature extracting and degradation status identification based on multiscale morphological decomposition spectral entropy," Journal of Vibration and Shock, vol. 32, no. 22, pp. 124-139, 2013.

[3] J. Yang and M. Zhao, "Fault diagnosis of traction motor bearings using modified bispectrum and empirical mode decomposition," Proceedings of the Chinese Society of Electrical Engineering, vol. 32, no. 18, pp. 116-122, 2012.

[4] P. Konar and P. Chattopadhyay, "Bearing fault detection of induction motor using wavelet and Support Vector Machines (SVMs)," Applied Soft Computing, vol. 11, no. 6, pp. 4203-4211, 2011.

[5] D. Zhu, Q. Gao, D. Sun, Y. Lu, and S. Peng, "A detection method for bearing faults using null space pursuit and S transform," Signal Processing, vol. 96, no. 5, pp. 80-89, 2014.

[6] S. Hong, Z. Zhou, E. Zio, and K. Hong, "Condition assessment for the performance degradation of bearing based on a combinatorial feature extraction method," Digital Signal Processing, vol. 27, pp. 159-166, 2014.

[7] B. Wang, H.-R. Li, Q.-H. Chen, and B.-H. Xu, "Rolling bearing performance degradative state recognition based on mathematical morphological fractal dimension and fuzzy center means," Acta Armamentarii, vol. 36, no. 10, pp. 1982-1990, 2015.

[8] H. Jiang, C. Li, and H. Li, "An improved EEMD with multiwavelet packet for rotating machinery multi-fault diagnosis," Mechanical Systems and Signal Processing, vol. 36, no. 2, pp. 225239, 2013.

[9] N. Mariyappa, S. Sengottuvel, R. Patel et al., "Denoising of multichannel MCG data by the combination of EEMD and ICA and its effect on the pseudo current density maps," Biomedical Signal Processing and Control, vol. 18, pp. 204-213, 2015.

[10] M. Žvokelj, S. Zupan, and I. Prebil, "EEMD-based multiscale ICA method for slewing bearing fault detection and diagnosis," Journal of Sound and Vibration, vol. 370, pp. 394-423, 2016.

[11] Y. Lei, Z. He, and Y. Zi, "EEMD method and WNN for fault diagnosis of locomotive roller bearings," Expert Systems with Applications, vol. 38, no. 6, pp. 7334-7341, 2011.

[12] H.-M. Dai, A.-Q. Xu, W.-F. Li, and W.-C. Sun, "Fault diagnosis of rolling bearings in mixed domain based on WMRMR," Journal of Vibration and Shock, vol. 34, no. 19, pp. 57-61, 2015.

[13] J.-B. Yu, "Bearing performance degradation assessment using locality preserving projections," Expert Systems with Applications, vol. 38, no. 6, pp. 7440-7450, 2011.
[14] F. Chen, B. Tang, T. Song, and L. Li, "Multi-fault diagnosis study on roller bearing based on multi-kernel support vector machine with chaotic particle swarm optimization," Measurement: Journal of the International Measurement Confederation, vol. 47, no. 1, pp. 576-590, 2014.

[15] Y. Lei, Z. He, and Y. Zi, "Fault diagnosis based on novel hybrid intelligent model," Chinese Journal of Mechanical Engineering, vol. 44, no. 7, pp. 112-117, 2008.

[16] J. Ben Ali, L. Saidi, A. Mouelhi, B. Chebel-Morello, and F. Fnaiech, "Linear feature selection and classification using PNN and SFAM neural networks for a nearly online diagnosis of bearing naturally progressing degradations," Engineering Applications of Artificial Intelligence, vol. 42, pp. 67-81, 2015.

[17] S. M. Vieira, J. M. Sousa, and U. Kaymak, "Fuzzy criteria for feature selection," Fuzzy Sets and Systems. An International Journal in Information Science and Engineering, vol. 189, pp. 118, 2012.

[18] S. Mitra, P. P. Kundu, and W. Pedrycz, "Feature selection using structural similarity," Information Sciences, vol. 198, pp. 48-61, 2012.

[19] X. Yao, X. D. Wang, Y. X. Zhang, and W. Quan, "Summary of feature selection algorithms," Control and Decision. Kongzhi yu Juece, vol. 27, no. 2, pp. 161-166, 192, 2012.

[20] T. Wang, M. Zhang, Q. Yu, and H. Zhang, "Comparing the applications of EMD and EEMD on time-frequency analysis of seismic signal," Journal of Applied Geophysics, vol. 83, no. 6, pp. 29-34, 2012.

[21] Z. H. Wu and N. E. Huang, "Ensemble empirical mode decomposition-a noise assisted data analysis method," Advances in Adaptive Data Analysis, vol. 1, no. 1, pp. 1-41, 2009.

[22] J. Dybała and R. Zimroz, "Rolling bearing diagnosing method based on empirical mode decomposition of machine vibration signal," Applied Acoustics, vol. 77, pp. 195-203, 2014.

[23] Y. Lei, "Machinery fault diagnosis based on improved HilbertHuang transform," Journal of Mechanical Engineering, vol. 47, no. 5, pp. 71-77, 2011.

[24] J.-D. Zheng, J.-S. Cheng, and Y. Yang, "A rolling bearing fault diagnosis method based on LCD and permutation entropy," Journal of Vibration, Measurement \& Diagnosis, vol. 34, no. 5, pp. 802-806, 2014.

[25] Y. Yang, H.-Y. Pan, and J.-S. Cheng, "The rolling bearing fault diagnosis method based on the feature selection and RRVPMCD," Journal of Vibration Engineering, vol. 27, no. 4, pp. 629-636, 2014.

[26] J. Xie, J. Lei, W. Xie et al., "Hybrid feature selection methods based on D-score and support vector machine," Journal of Computer Applications, vol. 31, no. 12, pp. 3292-3296, 2011.

[27] V. N. Vapnik, The Nature of Statistical Learning Theory, Springer, New York, NY, USA, 1995.

[28] K. A. Loparo, Case Western Reserve University Bearing Data Center. [EB/OL], http://csegroups.case.edu/bearingdatacenter/ pages/download-data-file.

[29] K. Zhu, X. Song, and D. Xue, "A roller bearing fault diagnosis method based on hierarchical entropy and support vector machine with particle swarm optimization algorithm," Measurement: Journal of the International Measurement Confederation, vol. 47, no. 1, pp. 669-675, 2014.

[30] Z. Liu, H. Cao, X. Chen, Z. He, and Z. Shen, "Multi-fault classification based on wavelet SVM with PSO algorithm to analyze vibration signals from rolling element bearings," Neurocomputing, vol. 99, pp. 399-410, 2013. 


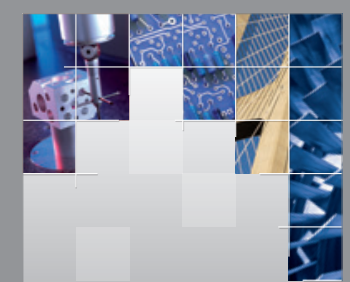

\section{Enfincering}
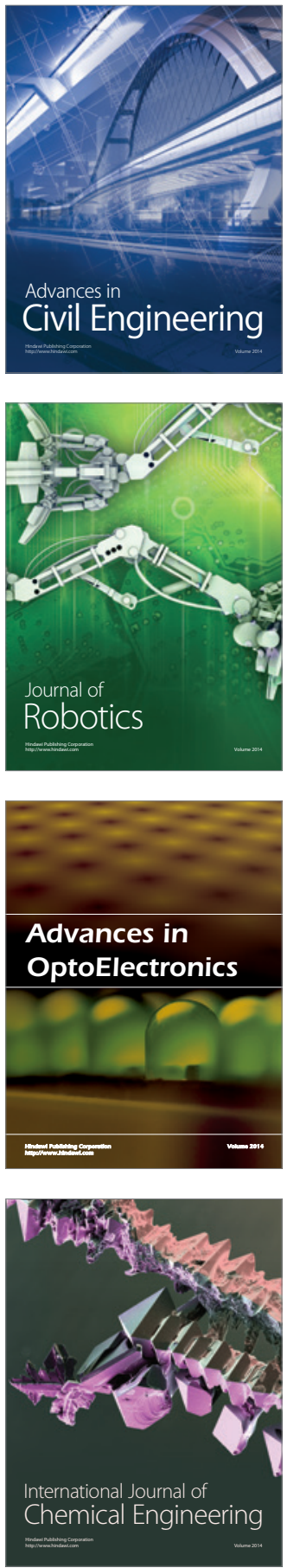

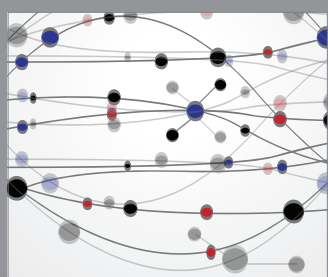

The Scientific World Journal

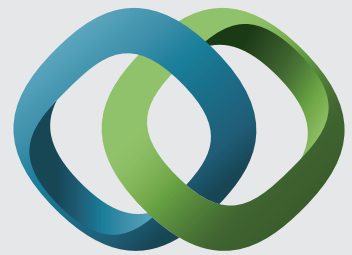

\section{Hindawi}

Submit your manuscripts at

https://www.hindawi.com
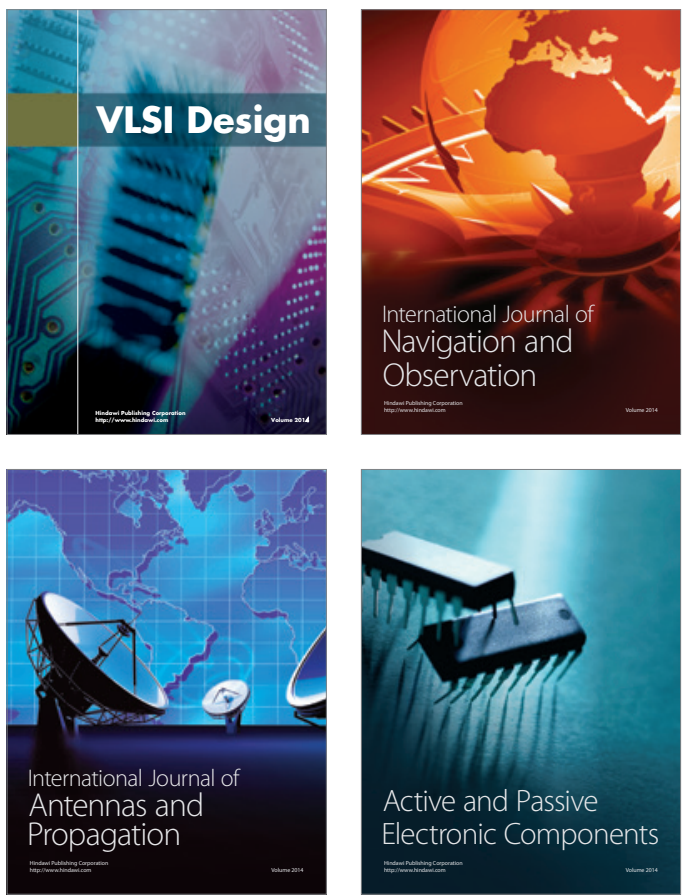
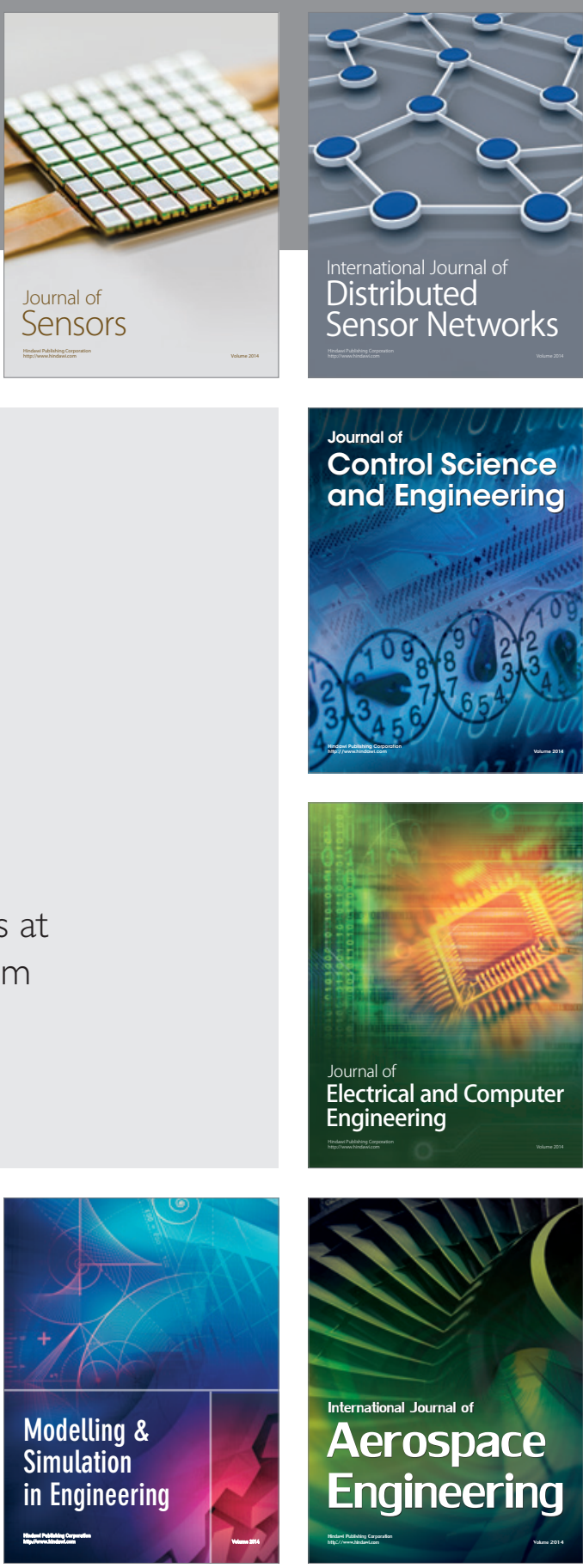

International Journal of

Distributed

Sensor Networks

$-$

Joumal of

Control Science

and Engineering
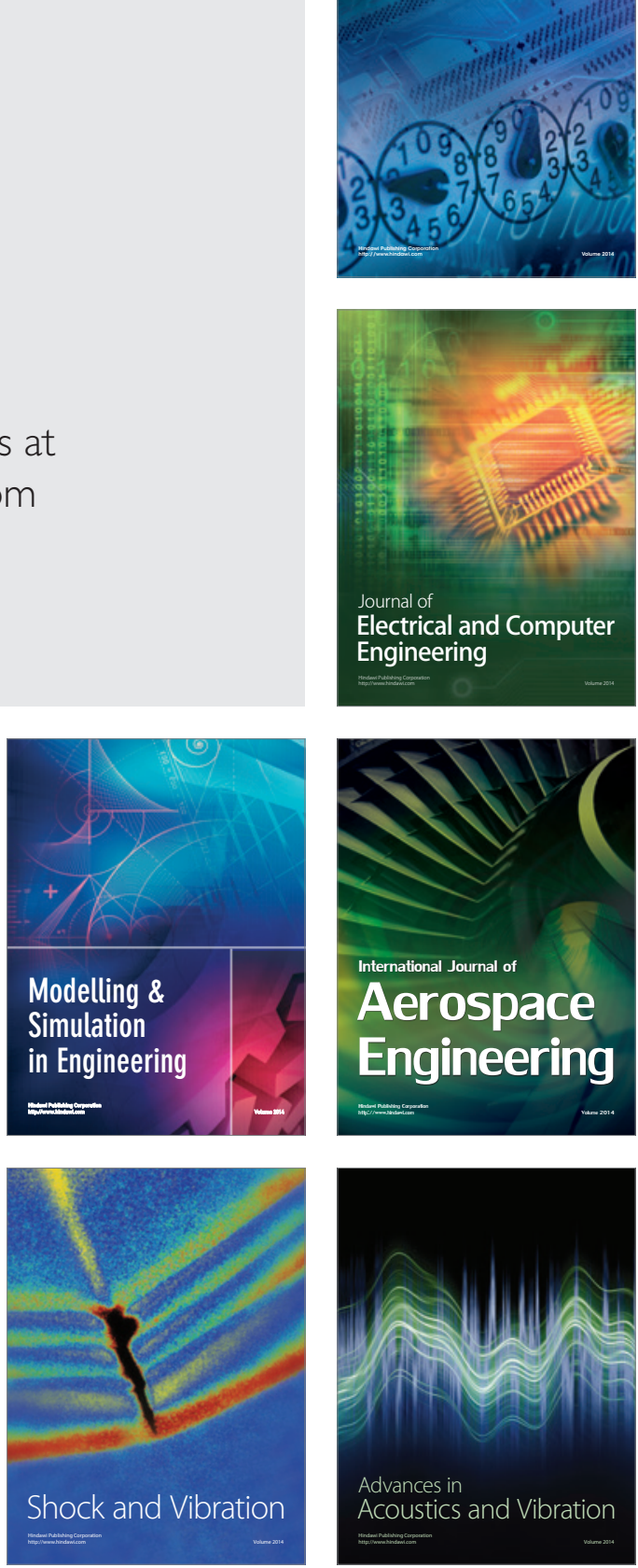